\title{
Desborda-da $^{1}$
}

\section{Overflowed}

\section{Marina Terrádez}

Correspondencia:

marinaterradez@hotmail.com
Filiaciones Institucionales:

Universidad Nacional de Rosario UNR (Argentina)

RESUMEN: Dificultades y complejidades en la práctica clínica, me convocan a interrogarme. Surgen algunas ideas a las que con entusiasmo doy lugar, las que a su vez se escapan de un camino lineal, y por tanto emergen intrincadas, rizomáticas.

Pacientes que relatan mucho malestar y sufrimiento vinculados a cierta persistencia e insistencia de algunos síntomas, ideas que los sumergen en un estado de mucha ansiedad y preocupación; angustia que por momentos desborda, un lazo transferencial complejo, intenso y ambivalente. Presos de respuestas impulsivas y demandas exigentes de resolución inmediata, se encuentran con dificultades para -entre otras cosas- lograr construir lazos que carezcan de fragilidad y labilidad.

Ciertos indicadores clínicos nos hacen pensar que opera la represión como mecanismo fundante, pero a la vez, se organizan manifestaciones que no condicen con una clásica neurosis de transferencia. Esto nos lleva a preguntarnos: $¿$ Es necesario pensar en nuevas posiciones diagnósticas?, $\mathrm{o}$ ¿ Son estas manifestaciones, novatas formas del malestar neurótico? Correlativo con esto: ¿Cuáles son las posibilidades y los límites para el trabajo analítico? En este trabajo, se propone interrogar algunos conceptos y designaciones vinculadas a estas nuevas modalidades de expresión del malestar en la cultura.

Palabras Claves: Bordes - Des-Bordes - Preguntas diagnósticas - Límites y posibilidades en la clínica

Cómo citar:

Terrádez, Mariana (2020).

Desborda-da en Revista Psicoanálisis en la Universidad N4. Rosario, Argentina, UNR Editora. Pág 119-130
ISSN: 2683-9938 (en línea)

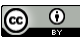

Licencia: Esta obra está bajo una Licencia Creative Commons Atribución-NoComercial-Compartirlgual 4.0 Internacional.

Responsabilidad editorial: Universidad Nacional de Rosario.

Argentina. Facultad de Psicología.
Recibido:

$27-03-2020$

Aceptado:

$09-06-2020$

Publicado:

$05-10-2020$ 
AвSTRACT: The difficulty and complexity in the clinical practice invite me to make questions about it. A few ideas arise and I allow them with enthusiasm, ideas which in turn escape a linear path and thus emerge as intricate and rhizomatic.

Patients that report a lot of discomfort and pain related to the persistence and insistence of certain symptoms, ideas that submerge patients in a state of much anxiety and worry; anguish that overflows at times, a transference bond that is complex, intense and ambiguous.

Ted to impulsive responses and strict demands for immediate resolution, they find themselves prone to difficulty for - among other things- being able to build up bonds that lack fragility and lability.

Certain clinical indicators make us believe that repression is the main mechanism, but at the same time, there are signs that do not coincide with a traditional transference neurosis. This leads us to ask ourselves: Is it necessary to think about new diagnostic positions?, or, Are these signs new forms of neurotic discomfort? Other questions are correlative to this: What are the possibilities and the limits for analytical work?

I suggest to question some concepts and diagnostic designations related to these new modalities of expressing discomfort in culture.

KEYwORDS: Borders - Overflows - Diagnostic Questions - Possibilities and limits in the clinic. 


\section{INTRODUCCIÓN}

Me voy me voy

Ya no puedo estar acá

No aguanto este desastre

Y vos, y vos

No aprendiste a controlar

Esos momentos de desborde

Ya no puedo más así

No lo ves

No me queda mucho más que decir

(...) Esperé tanto que al fin

disparé

(Fragmento de "Culpables"

de Airbag)

Las dificultades y la complejidad en la práctica clínica, me convocan a construir algunos interrogantes que escapan al pensamiento lineal, emergiendo en sus formas más intrincadas y rizomáticas, a las que entusiasmadamente doy lugar.

En este sentido pienso la experiencia analítica con una paciente a la que llamaré Ana Clara. Esta mujer joven, de aspecto aniñado, me consulta a partir de la derivación de su anterior analista. Se presenta manifestando tener livianas dificultades acompañadas de algún malestar, situación que contrasta con un mensaje que recibo a la semana siguiente de la primera entrevista. Era una persona allegada a su entorno, quien se ofrece brindar ayuda frente a cualquier situación en que yo considere que ella pudiera necesitarlo. Es entonces cuando además de mi sorpresa, aparecen las primeras preguntas. Tempranamente, se fue planteando un escenario analítico, en el que todas las señales indicaban que no sería una experiencia común, situación que confirmé a la brevedad.

Rápidamente diversas cuestiones comenzaron a florecer abonadas de mucho malestar y sufrimiento: cierta persistencia e insistencia de algunos síntomas, ideas recurrentes que la sumergían en un estado de mucha ansiedad y preocupación; angustia que por momentos desborda, un lazo transferencial muy complejo, intenso y ambivalente; extrema "porosidad" (Sternbach, 2016) al devenir de los acontecimientos cotidianos, por lo que su vida y sentir oscilan vertiginosamente de la alegría a la más profunda tristeza, frente a situaciones que podrían juzgarse como nimiedades o contratiempos. Presa de respuestas impulsivas y demandas que se presentan con exigencia de inmediatez le dificultan -entre muchas otras cosasconstruir lazos sólidos y perdurables en el tiempo.

En ocasiones relata algunos fragmentos de su historia, en otras, se defiende diciendo que hay muchas cosas que le han causado dolor, que ya las ha "trabajado con otros profesionales" negándose a hablar, por momentos "muestra" que quiere ocultar cosas y si bien relata sueños, y realiza algunas asociaciones, también suelen aparecer silencios que delatan un vacío, como si se interrumpiera la cadena discursiva. No deja de presentarse la emergencia de una angustia, que brota no como señal, sino de forma masiva, seguido de la desesperanza absoluta, situación que emparento conjuntamente con otros acontecimientos de su historia vinculados a algunas escenas de intento de suicidio, e internaciones ocurridas en algunas oportunidades.

Ana clara revela ciertos indicadores clínicos que nos hacen pensar que opera la represión como mecanismo fundante, no hay alteraciones del lenguaje ni otros signos que puedan darnos a pensar en una psicosis, pero a la vez, organiza manifes- 
taciones que no condicen con una clásica neurosis de transferencia. Esto nos lleva a preguntarnos: ¿Es necesario pensar en nuevas posiciones diagnósticas?, o ¿Son estas manifestaciones, modos en los que el malestar neurótico adquiere novatas formas en la actualidad? Correlativo con esto: ¿Cuáles son las posibilidades y los límites para el trabajo analítico?

\section{LA TEORÍA, NUESTRO MARCO}

Sabemos de la importancia de la formalización de conceptos en tanto funcionan como operadores clínicos, de hecho, nuestra práctica está atravesada y marcada por la teoría sobre la cual nos apoyamos. Pensado de este modo, el marco teórico funciona como un sostén, en el sentido de un punto de apoyo imprescindible, marco a partir del que podemos pensar. De todas maneras, esto no debería conducirnos a la negativa de poder preguntarnos y cuestionar esa misma estructura que devino andamiaje. En el libro Tramas Teoría, clínica y ficciones para un psicoanálisis contemporáneo, Susana Sternbach se refiere a la idea de concepto en su raíz alemana como derivado de "garra" y en ese sentido, es solidaria también de la de "predador" definiéndolo como "un tirano que en aras de la solidez deja de lado la polivalencia de las palabras y lo inaprensible del humano en singular" (2016, P. 51)

El concepto y por extensión, la teoría, requieren establecer generalidades que funcionan como un encuadre, se constituyen en una referencia y es necesario que así acontezca. Por consecuencia, la singularidad queda elidida, y así la teoría se distancia del caso por caso, no le da lugar. Sin embargo, no ocurre lo mismo al revés, donde en el trabajo con cada paciente podemos pensar y es necesario una referencia a la teoría.

De todas maneras, sostengo que en ocasiones habría cierta dificultad, que radica en considerársela como un "baluarte exhaustivo" (Sternbach, 2016, p. 51) por lo que se intenta ajustar todo a ese modelo, sin cuestionar ni interpelar nada del edificio conceptual.

La idea que intento transmitir es que, si el concepto trata de ceñir, de amarrar o apresar una idea para articularla en una lógica que corresponde a la teoría, en nuestra práctica debemos pensar con los conceptos, interrogarlos, ponerlos a jugar, alivianarlos, para poder evitar quedar atrapados por las garras de la fijeza y entre otras cosas, realizar nuevas articulaciones posibles que puedan también hacer teoría.

\section{¿Nuevos diagnósticos? o ¿Nuevas MODALIDADES DE LA NEUROSIS DE TRANSFERENCIA?}

Hace algunos años venimos escuchando a diversos autores hablar de trastornos narcisistas no psicóticos, estructuras borderline, personalidades fronterizas, patologías del acting out, personalidades como sí, entre otras designaciones posibles para referirse a pacientes que manifiestan -en términos generales- dificultades como las que mencioné anteriormente. Asimismo, como contracara de esto, algunos analistas manifiestan una posición controversial respecto de estos desarrollos. Si bien son amplias y variadas las argumentaciones en su contra, entiendo que uno de los puntos centrales, radica en que no se ajustan a lo que se ha entendido como la clásica tripartita divisoria diagnóstica realizada 
por Lacan conocida como las "estructuras clínicas” neurótica, psicótica y perversa. En este sentido, consideran estas manifestaciones como nuevas modalidades que adquieren ciertos fenómenos en este contexto histórico social.

Así, la idea de estructuras clínicas, funcionaría como lo que da consistencia a los casos, organizándose un esquema que opera de manera paralela a la de los clásicos cuadros nosográficos de la psiquiatría, lo que se podría designar como una " $p s i$ quiatrización del psicoanálisis” (Kreszes, 1994 citado en Muñoz 2014 p. 28). Entiendo, es también la búsqueda de cierta garantía clínica.

Por otro lado, tampoco entiendo que el problema se resuelva con la idea de pensarlo como mixturas tal como lo indican las consideraciones de algunas líneas del psicoanálisis anglosajón, al modo de núcleos o fragmentos psicóticos en estructuras neuróticas o al revés, ni de pasajes de un modo a otro como podría ser la idea de un continuo clínico, que marcara sucesiones desde la posición neurótica a la psicótica y viceversa.

La última cuestión que es importante considerar, reside en suponer que deba organizarse una mejor clasificación, entendiéndose mejor como "más completa" para dar cuenta de manifestaciones que no entran en lo ya establecido, como si pudiera saturarse la hiancia estructural, y el conjunto pudiera completarse.

Sin corrernos del problema, Pablo $\mathrm{Mu}$ ñoz (2014) realiza un breve recorrido por desarrollos teóricos de otros analistas/ autores quienes, frente a este dilema, vale aclarar, de casos clínicos complejos que no se ajustan ni asemejan a una "clínica común”, elaboran lecturas diferentes.
Por su parte, toma las consideraciones de Jean-Claude Maleval, quien plantea que, en la actualidad, los analistas se enfrentan a la demanda de sujetos que presentarían ciertos modos de funcionamiento solidario de la psicosis, pero carentes de fenómenos que pudieran confirmar ese diagnóstico, por tratarse de neurosis, más allá de lo cual, sugiere considerar que estas expresiones clínicas se vincularían con algunas condiciones particulares, relacionadas al modo de alojamiento del nudo en la estructura del sujeto, tales como lo que cito a continuación: "indicios de la no extracción del objeto a, aflojamiento del punto de capitonado, prevalencia de las identificaciones imaginarias" (Maleval en Muñoz, 2014, p. 53). Cabe destacar, que hablar del aflojamiento del nudo, no sería su desarticulación, ni su desanudamiento, por lo que considera una organización neurótica que sufre ciertos incidentes, lo que organizaría -si vale la expresión- una estructura más lábil.

Por su parte, Jean-Jacques Rassial, nos hablará sobre la categoría de "estado límite” (Muñoz, 2014 p. 54) lo que permite diferenciarlo de un sujeto en "situación limite”. De esta manera, el autor, permite pensar a sujetos neuróticos que pueden estar al borde de la desorganización en el modo en que manifiestan su malestar, en tanto, a la vez se vincula con representaciones relacionadas a la fragilidad, debilidad, complicaciones narcisistas y un acortamiento de la distancia entre interior-exterior, entre otros.

Sin desviar del asunto, Muñoz (2014) sostiene que el término "estructuras clínicas" no es un sintagma que haya sido plasmado por Lacan en sus escritos, ni del que haya hablado en sus seminarios, 
en cambio, sí se ha referido a "estructuras freudianas", con un sentido diferente al que comúnmente se le da al primero.

El autor, retoma las distinciones de neurosis, psicosis y perversión, a partir de considerar al sujeto como efecto de la estructura significante y discursiva, realizando una torsión. De este modo, Muñoz referirá: “(...) con el fin de revisar sus fenómenos y atendiendo a la premisa de pensar al sujeto en cuanto determinado por la estructura del significante (...), los trata como "tipos clínicos" (2014, p. 35). En este sentido entiende la noción de tipo, como refiriéndose a la idea de modelo, de ejemplar, en tanto singular, diferente de la de estructura, que subsume a la cuestión de universalidad.

En virtud de esta idea, y a la vez retomando nuestro tema central, cabe destacar que Muñoz, valiéndose de la noción lacaniana de sujeto del inconsciente, es decir, que el sujeto es un efecto de discurso cuya emergencia ocurre a partir de lapsus, equívocos, sueños, etc. según la estructura de la lógica formal que se vale de la metáfora y metonimia para la sustitución significante, hablará de "suspensión de la neurosis” (Muñoz, 2014, p. 154) Se referirá de este modo, a ciertas manifestaciones que adoptan modalidades que comportan la detención de los movimientos subjetivos que le son propios a la estructura neurótica del síntoma en la articulación de la cadena discursiva.

Relaciona este fenómeno de suspensión de la neurosis con el mecanismo de la Verwerfung, no en el sentido de la forclusión del Nombre-del-Padre, propio de la psicosis, sino en relación a un rechazo de la simbolización de sucesos traumáticos, de ciertos accidentes en el devenir del sujeto.
"El sujeto desarrolla en su discurso analítico su verdad, su integración, su historia. Pero en esa historia hay huecos: allí donde se produjo lo que fue Verwerfen (...) es decir, un rechazo originario" (Lacan, 1953-1954 / 1996, p 411). En este sentido, la cita de Lacan, se puede entender en relación a que ciertos elementos del orden de lo imaginario y lo real que no han sido integrados simbólicamente, podrían ser elididos en la cadena significante, no obstante, una posibilidad de su admisión sería por la vía del análisis.

Muñoz, entonces, plantea que hablar de neurosis, psicosis, o cualquier otra, no indica la esencia de un sujeto, sino "nombres de cierto tipo de efectos de lenguaje en el hablanteser" (2014, p. 59). A partir de esta idea, el autor construye lo que podría considerarse como las neurosis en suspenso a saber: que puede haber cierta discontinuidad, en el sentido de que algunos efectos tienen incidencia en las características y condiciones de la estructura del lenguaje, implicando cortes o rupturas del continuum de la cadena. Habría algo del orden del fenómeno que se presenta, que aparece o irrumpe como sorpresa. En este contexto, me parece fundamental considerar la idea de fenómeno, como una manifestación del malestar psíquico que no se condice con la formación de compromiso propia del síntoma neurótico. Ello implica desestimar que lo que emerge o irrumpe sea necesariamente una formación del inconsciente, más bien debemos en ocasiones suponer el desarrollo de conformaciones que según Muñoz, constituyen “configuraciones sintomáticas que comportan que la estructura no opera o bien está suspendida: neurosis en stand by." (Muñoz, 2014 p. 151) 
Tonalizado por signos que podrían considerarse como una histeria grave, pero también por la insistencia de determinadas manifestaciones que resultan tener algunas particularidades, especialmente porque no condicen con una neurosis de transferencia, la experiencia con Ana Clara, correlativa de una época determinada, discurre con nuevas formas y mecanismos que le son propios, y a la vez comunes a muchos otros pacientes con los que trabajamos hoy día.

Para complejizar aún más el panorama, autores como Silvia Amigo y Héctor Yankelevich, consideran que este tipo de manifestaciones clínicas, corresponden a fenómenos que denominan neurosis narcisistas. Distanciándose del uso freudiano del término que las emparenta directamente con la hebefrenia y la melancolía, le dan una nueva significación o especificidad.

Antes de introducir sus ideas, creo pertinente e importante destacar que la expresión neurosis narcisista exige en principio contemplar dos cuestiones:

Por un lado, hay una organización solidaria del modo neurótico, lo que supone que aplican mecanismos propios de este tipo clínico. Por otro lado, en tanto se refiere al narcisismo, entendemos que, en la operatoria de estructuración del psiquismo, esos procesos revisten de ciertas vicisitudes que presentan consecuencias narcisistas.

En 1914, cuando Freud escribe Introducción al narcisismo, formaliza por primera vez este término ya utilizado anteriormente (Sobre el caso Schreber -1911-) refiriéndose a él a partir de las catexias libidinales. El narcisismo implica que la libido toma al yo como objeto de amor, saciando o satisfaciéndose en él como ha- bitualmente lo haría con el objeto. El descubrimiento freudiano, es que este proceso en su mecanismo constitutivo, ocurre conforme también con "un nuevo acto psíquico” (Freud, 1968-1914), la identificación, que permitiría la instauración del Yo.

Sin profundizar en detalles, sabemos que la organización del narcisismo solidaria de la estructuración del yo en ciernes requiere de la concatenación lógica de procesos que deben acontecer. Ahora bien, si las neurosis narcisistas presentan determinadas particularidades, debemos pensar que existen posibles avatares que pueden ocurrir e intervenir en su organización. ¿Cuáles son?, ¿Cómo es que se produce su manufactura?, ¿Qué posición del Otro puede favorecer el modo narcisista en la neurosis?

En el seminario La Relación de objeto, Lacan se refiere a la castración, y sus efectos, planteando una situación que puede sobrevenir vinculada a su operatoria:

es en tanto que el complejo de castración ha sido, a la vez, franqueado, pero sin poder ser plenamente asumido por el sujeto, que se produce una identificación con una suerte de imagen bruta del padre, imagen que sostiene los reflejos de sus particularidades reales en lo que éstas tienen de pesado, y aún de aplastante. Vemos aquí una vez más renovarse el mecanismo de reaparición en lo real, pero esta vez de un real en el límite de lo psíquico, en el interior de las fronteras del yo -de un real que se impone al sujeto de un modo casi alucinatorio, en la medida en que ese sujeto, en un momento dado, abandona la integración simbólica del proceso de castración. (Lacan, 1994 p. 419) 
Cabe señalar, siguiendo la lectura realizada por Héctor Yankelevich, que esta cita no refiere a un retorno de lo forcluido en lo real como ocurriría en la psicosis, sino que remite a la idea de que habría una renegación o rechazo que, como mecanismo defensivo, implicará un retorno en lo real que se impone dentro de los límites del yo. Así podemos decir también, que el yo incluye un real que por momentos se le impone, porque hay una pérdida de la referencia simbólica. Es entonces, esta dificultad la que genera un malestar que por momentos se vuelve intolerable, goce que se destaca en parte por su opacidad, y también por el efecto de exclusión de sentido. En palabras de Yankelevich:

una astilla de lo real no deja nunca al yo en paz, satisfaciéndose en él, sin que podamos darnos la facilidad de considerar a este real sólo como un resto de goce que reclama Encore! El goce del que se trata aquí, en el que el sujeto se debate en las neurosis narcisistas, ejerce su efecto sobre la cadena inconsciente, dificultando la creación de significantes por metáfora y metonimia, empobreciendo el investimento de recuerdos (...) (Yankelevich, 2016, parr. 14)

El autor, sostiene a la vez, que los avatares en la asunción de la castración, se vinculan con el hecho de que esta operatoria ha acontecido tempranamente en relación a las posibilidades del sujeto para sostenerse en relación al objeto libidinal. Esto haría referencias a que “ (...) hubo una anticipación forzada del sujeto en el trabajo de la separación con el Otro, sin poder permitirse el tiempo de un período de alienación suficiente (...)" (Yankelevich, 2017, pp. 71-72).
Sería importante destacar que, si bien entendemos que estos sucesos en parte determinan este modo de padecimiento narcisista, también se destaca cierta posición del Otro que favorecería estas condiciones , al obligar o propiciar que el sujeto ofrezca su yo, como objeto separador.

Retomo varias cuestiones: la castración vivida de manera anticipada, da cuenta de una relación de apuntalamiento invertida entre el sujeto y el Otro, lo que quebranta las posibilidades del mismo para apoyarse y sostenerse en un lazo amoroso. Así, según el decir de Yankelevich “(...) es el rechazo - y no frustración- de amor lo que estará en el origen de la más o menos gran aptitud de ciertos sujetos para sentir y ser psíquicamente lesionados por eventos que, para ellos, devienen traumáticos” (Yankelevich, 2017, p. 73) Este trauma, nominad como falta de amor (Yankelevich, 2017) puede ser considerado de dos maneras: como un rechazo de amor en el sentido de ciertas dificultades en el lazo libidinal del Otro, así como de un amar que no ha podido penetrar ni arraigarse a la vida sexual infantil de la madre, en donde el niño sólo es amado en la medida en que funciona como un espejo que refleja una imagen a través de la cual ella puede amarse a sí misma. Así el sujeto deviene en objeto de apuntalamiento narcisista, en el cual, insistimos, el esquema queda invertido.

Siguiendo esta línea, en concordancia con lo planteado por el autor, en "Paradojas clínicas de la vida y la muerte" Silvia Amigo hace referencia a la metáfora de la díada madre-hijo, planteando dos situaciones: Por un lado, la madre, que gracias a su capacidad de holding (Winnicott) puede sostener a su hijo, al que inviste fálicamente, aun aceptando la falta, y por tanto sin que el niño devenga sutura de 
su propio narcisismo. Situación estructural que podríamos pensar como la mejor opción, siempre que ninguna contingencia desvíe del camino marcado.

Por otro lado, relata otra situación donde la madre, ubicando al niño en la posición de objeto de su propio juego, se transforma en instrumento del sostén materno, el que deviene en "esclavo", prevaleciendo una relación de objeto (la que él es para el Otro) que tenderá a repetirse incesantemente. "En esta situación, emparentada a la melancolía pero de factura neurótica, la sombra del objeto cae sobre el yo" (Amigo, 2009, p. 93.)

¿Quién sostiene a Ana Clara? Su nombre es homofónico al de su madre, sonidos pegoteados, demasiado cercanos, al punto que por momentos aparecen como indiferenciados. Desde pequeña, Ana tiene que sostener a una madre depresiva, que demanda imperativamente, sin intermediación de un padre también afectado por profundas dificultades, que por momentos no puede más que ausentarse. En su adolescencia se sitúan sus primeras crisis, donde ella también reclama sostén, para eso, pareciera es condición tener que deprimirse, y la muerte, le aparece como idea de la única opción posible para separarse del Otro.

Retomando estos desarrollos, cabe preguntarse: los mecanismos y el modo sufriente que Ana Clara manifiesta ¿representa procesos propios de lo que destacaríamos como neurosis narcisistas?, o ¿debemos leerlos como fenómenos que disrumpen la continuidad de la neurosis, al modo planteado como neurosis en stand by?

\section{El IMPERATIVO Superyoico}

Continuando con lo que veníamos planteando, cabe detenerse en algunas cuestiones. Habíamos mencionado que habría un núcleo de real imposible de extirpar que se constituirá en el nudo del Superyo, el que devendrá en el mayor enemigo íntimo, principal objetor que denunciará con saña y desprecio cada vez que haya fracaso en encarnar el ideal, porque todo desajuste que imposibilite suturar la demanda del Otro se constituirá en una "mancha" (Amigo, 2009, p. 101) que estorba y que pretende reiteradamente ser eliminada, insistencia que sólo encuentra el fracaso. Esto nos indica también, que si bien siguiendo a Freud, el Super Yo se constituye como "heredero del complejo de Edipo", su materia prima, tiene trazas más arcaicas.

Cuando en el sujeto prima la lógica de la transmisión del líder de masas y no la del padre, la exterminación se deja oír no sólo en su cara exterior, como violencia concentracionaria. También hay un correlato intrapsíquico de esa violencia (...) El objeto, humillado, (...) deseado en exterminio, toma su revancha $(. .$.$) y bajo$ sus órdenes, (...) deja oír su imperativo ¡Goza!. El yo estará siempre por debajo del nivel que le exige esta instancia crítica (el super yo) y un malestar bastante mayor que el ineliminable malestar cultural se dejará sentir. (Amigo, 2009, p. 129)

Podemos afirmar entonces, como ya lo venimos planteando, que algo ha fallado en la operatoria simbólica, por lo que se dificulta refrenar la desmesura de la des- 
carga pulsional directa, así como el reconocimiento de la alteridad. La terceridad asoma sin eficacia para dar lugar al deseo, que adviene frágilmente, el profundo malestar se pone en acto de manera destructiva, el Super Yo se erige fuertemente en su versión más tanática, insensato y cruel que en Ana Clara aparece bajo diversas expresiones y manifestaciones. Subsidiaria de esta idea, a veces dice ser "un volcán que desborda”. ¿Qué es lo que hace erupción? ¿Qué provoca su desmesura? El intenso sufrimiento que siente por no conseguir las cosas que se propone, de la forma en que se las propone, en el momento en que lo dispone, por sentirse menos que cuanta persona hay alrededor suyo, en tanto considera que tienen logros cuando ella siente que nunca puede arrancar, por las ideas de muerte que aparecen insistentemente como única salida.

\section{El trabajo Del anAlista}

Ana Clara sitúa al analista en una posición compleja: además de establecer un lazo transferencial cargado de ambivalencia, la posibilidad de trabajo cambia de condiciones de sesión a sesión.

En algunas oportunidades, como se deslizó anteriormente, relata sueños, se dispone a asociar, hay riqueza metafórica, pero en otras, cualquier intervención que permita operar en el sentido de la división subjetiva queda obturada, anulada como si rechazara por momentos el reconocimiento del inconsciente. No me refiero a la negación en el sentido de la defensa freudiana, sino más bien un rechazo más arcaico, que remite a expulsar lo propio, apareciéndose como ajenidad. En esas ocasiones cualquier intervención que señale la emergencia del sujeto en el discurso se vive como una amenaza, de la que se defiende abroqueladamente. Me pregunto: ¿no responder con el yo, implica dejar de ser ese objeto que sostiene al Otro?, ¿Correrse de ese lugar, es sentirse asomar al precipicio?

Por momentos, Ana Clara ha estado en el abismo, amenazas, ideas, e "intentos" de suicidio la han acompañado desde su juventud, regadas o sostenidas de una angustia que no encuentra el freno. En los momentos, en que se manifiesta como una escalada que se incrementa positivamente, el recurso ha sido acompañarla, para donar palabras que pudieran bordearla, al modo de hacer una marca que pudiera funcionar como un punto de capitoné, que pudiera anudar algo a esto que discurre volcánicamente, metonímicamente. Incluso, en muchas ocasiones el poder delimitar o situar que podríamos hablar en determinado momento del día, funcionaba también haciendo de linde posible.

Han sido muchos los días, en que el trabajo con Ana Clara resultó muy dificultoso, en el sentido de que la analista continuara muy preocupada por ella, al finalizar las sesiones. La observación de cierta reticencia a los efectos de un análisis en el sentido de que, por momentos, la intervención a través de la interpretación no hace mella nos hace preguntarnos sobre cuál es el lugar del analista en la dirección de la cura. Resulta evidente que aquí, nuestra labor -muchas veces- no se sostiene en relación a las formaciones del inconsciente.

(...) con pacientes que consideran que deben ser únicos, no siendo ese "único" el resultado de una forclusión psicotizante, sino una vía ascendente hacia Un significante del que esperan obtener estatura 
humana, ya que, de no ser esta tentativa desesperada, serían sólo desecho. Es sólo el cumplimiento de esa tarea ímproba lo que los devolverá al "uno entre todos" de la sociedad humana, pero redimidos de la falta moral, realmente cometida o que en su fuero íntimo adjudican a uno de sus padres o a los dos por igual (Yankelevich, 2016, parr. 21)

Lacan (1955) sostenía que no hay que retroceder frente la psicosis, a lo que agrego, ni ante cualquier experiencia clínica por más dificultad que ella revista, siempre que nos convoque el deseo como analistas. Más allá de esto, en casos como este, me resulta pertinente pensar que en el sujeto habrá un intenso malestar que, por momentos, disruptivamente se presentará siempre. Aún y en ese caso, nuestro oficio, quizás se limite a encontrar un modo posible de acompañarlos. La incesante búsqueda por separarse del Otro, que deviene en permanente batalla para la que carecen de armas, ubica al analista en una tarea compleja, labor para la que tendrá que disponerse a leer las estrategias de un juego peligroso que oscila de forma constante, entre el abandono de la experiencia analítica y diversos actos en los que se coquetea con la muerte. "Si fuera el caso, la vida toda sería un tratado del combate en vano". (Yankelevich, 2019, P. 192)

\section{Notas:}

1. El juego de palabras presente en "Desborda-da", remite a la escansión del Fort y el Da, alternancia que se sostiene sobre la dimensión temporal, en la que la presencia se organiza sobre una ausencia posible. Así el Fort-Da sirve para pensar la constitución del sujeto a partir de las operaciones de alienación y separación.

\section{REFERENCIAS BIBLIOGRÁFICAS.}

Amigo, S. (2009) Paradojas clínicas de la vida y la muerte. Rosario, Argentina. Biblioteca Nueva.

Freud, S. (1911) Observaciones psicoanalíticas de un caso de paranoia (dementia paranoides) autobiográficamente descrito. En Obras completas. Bs AsMadrid. Biblioteca Nueva. Tomo II (pp.879-891)

Freud, S. (1914) Introducción al narcisismo. En Obras completas. Bs As- Madrid. Biblioteca Nueva. Tomo I (pp. 1083-1096)

Freud, S. (1915) Pulsiones y sus destinos. En Obras completas. Bs As- Madrid. Biblioteca Nueva. Tomo I (pp, 10351045

Freud, S. (1920) Más allá del principio de placer. En Obras completas. Bs AsMadrid. Biblioteca Nueva, Tomo I (pp, 1097-1127).

Freud, S. (1923) El yo y el ello En Obras completas. Bs As- Madrid. Biblioteca Nueva. Tomo II (pp.9-31) 
Freud, S. (1924) Neurosis y psicosis. En Obras completas (pp.499-501) Tomo II Ed. Bs As- Madrid. Biblioteca Nueva. Tomo II

Lacan, J. (1975) Seminario 1. Los escritos técnicos de Freud. Buenos Aires, Argentina. Ediciones Paidós.

Lacan, J. (1981) Seminario 3. Los escritos técnicos de Freud. Buenos Aires, Argentina. Ediciones Paidós.

Lacan, J. (1994) Seminario 4. La relación de objeto. Buenos Aires, Argentina. Ediciones Paidós.

Lacan, J. (2004) Seminario 10. La Angustia. Buenos Aires, Argentina. Ediciones Paidós.

Lacan, J. (1994) Seminario 20. Aún. Buenos Aires, Argentina. Ediciones Paidós

Muñoz, P. (2014). Las locuras según Lacan. Consecuencias clínicas, éticas y psicopatológicas. Buenos Aires, Argentina. Letra Viva

Nasio, David (2017) Los gritos del cuerpo Buenos Aires, Argentina. Paidós.

Sternbach, S (2016) Tramas. Teoría, clínica y ficciones para un psicoanálisis contemporáneo. Buenos Aires, Argentina. Letra Viva.

Yankelevich, H (2016) Las neurosis narcisistas y el trauma no sexual. Diario literario digital.

Yankelevich, H (2017) “Trauma, muerte y sexualidad. Para (re)introducir las neurosis narcisistas". Revista Psicoanálisis y Hospital. Buenos Aires, Argentina. Ediciones del Seminario. Número 51 (pp, 57-76).

Yankelevich, H (2019) Ensayos sobre autismo y psicosis. Buenos Aires, Argentina. Cascada de letras.

\section{Marina Terrádez:}

Psicóloga, Psicoanalista. Profesora Adjunta de la cátedra Biología Humana de la Facultad de Psicología (UNR). Profesora Titular de la carrera de especialización en kinesiología y fisioterapia neurológica en Universidad de Gran Rosario. 